\title{
Pathogenic LRRK2 R1441C mutation is associated with striatal alterations
}

Harry S. Xenias ${ }^{1}$, Chuyu Chen ${ }^{2 *}$, Shuo Kang ${ }^{2 *}$, Suraj Cherian ${ }^{1}$, Xiaolei Situ², Bharanidharan Shanmugasundaram², Giuseppe Scesa², C. Savio Chan ${ }^{1 \#, ~ L o u k i a ~ P a r i s i a d o u # ~}$

${ }^{1}$ Department of Physiology, Feinberg School of Medicine, Northwestern University, Chicago, IL, USA

${ }^{2}$ Department of Pharmacology, Feinberg School of Medicine, Northwestern University, Chicago, IL, USA

*equal contribution

${ }^{*}$ corresponding authors

Correspondence should be addressed to C. Savio Chan at saviochan@gmail.com or Loukia Parisiadou at loukia.parisiadou@northwestern.edu

Running title: Striatal alterations in LRRK2 knock-ins

\section{Acknowledgments}

This work was supported by Michael J. Fox Foundation for Parkinson's Research (LP), NIH R01 NS097901 (LP), R01 NS097901 (CSC), R01 NS069777 (CSC), P50 NS047085 (CSC), R01 MH112768 (CSC), R01 MH109466 (CSC), R01 NS088528 (CSC), T32 NS041234 (HSX), F32 NS098793 (HSX). We thank Brianna Berceau for colony management and technical support. We also thank Dr. Heather Melrose for providing the LRRK2 G2019S knockin mice. 


\section{Author contributions}

HSX and SC conducted the electrophysiological measurements. HSX designed and conducted the voltammetric measurements. CC, SK, BS, XS, and GS conducted behavioral testing. HSX and SK conducted statistical analysis. LP, CSC, and HSX wrote the manuscript with input from all co-authors. LP and CSC designed, directed, and supervised the project. All authors reviewed and edited the manuscript. 


\section{Abstract (136 words)}

LRRK2 mutations are associated with both familial and sporadic forms of Parkinson's disease (PD). Convergent evidence suggests that LRRK2 plays critical roles in regulating striatal function. Here, by using knock-in mouse lines that express the two most common LRRK2 pathogenic mutations-G2019S and R1441C-we investigated

5 how pathogenic LRRK2 mutations altered striatal physiology. We found that R1441C mice displayed a reduced nigrostriatal dopamine release and hypoexcitability in indirect-pathway striatal projection neurons. These alterations were associated with an impaired striatal-dependent motor learning. This deficit in motor learning was rescued following the subchronic administration of the LRRK2 kinase inhibitor Mli-2. In contrast, though a decreased release of dopamine was observed in the G2019S knock-in mice no concomitant cellular and behavioral 10 alterations were found. In summary, our data argue that the impact of LRRK2 mutations cannot be simply generalized. Our findings offer mechanistic insights for devising treatment strategies for PD patients.

\section{Introduction (321 words)}

The identification of LRRK2 mutations provided important insights into the genetic basis of Parkinson's disease (PD). Autosomal dominant mutations in LRRK2 are the most common genetic cause of late-onset PD to date $^{[1,2]}$. Accordingly, compelling evidence from genome-wide association studies has identified LRRK2 as a risk factor for sporadic PD ${ }^{[3-5]}$. Patients with LRRK2 mutations exhibit clinical and pathological phenotypes that are indistinguishable from sporadic PD ${ }^{[6-8]}$, suggesting common disease mechanisms. These findings urge further

20 efforts in understanding mutant LRRK2-induced pathophysiology. The yielded knowledge about the impact of different LRRK2 mutations can be leveraged for therapeutic benefit in PD.

The LRRK2 mutations G2019S and R1441C (hereafter referred to as GS or RC) are commonly found in familial PD ${ }^{[9-11]}$. These two mutations are located in the kinase and ROC (Ras of complex GTPase) domains of the LRRK2 protein, respectively (Figure 1a). It is widely thought that all pathogenic mutations increase LRRK2 kinase 25 activity ${ }^{[12,13]}$. Recent studies show that the RC and other related mutations such as $\mathrm{R} 1441 \mathrm{G} / \mathrm{H}$ result in even greater activation of LRRK2 kinase activity compared to GS mutation ${ }^{[1,13-15]}$. Though the literature focuses on the 
GS mutation, this highlights the importance of determining the impact associated with the RC mutation. The yielded knowledge should, in turn, facilitate the development of therapeutic interventions. Relatedly, previous studies provide support for distinct clinical features associated with different $L R R K 2$ variants ${ }^{[16,17]}$.

Transgenic mouse models overexpressing LRRK2 GS and RC mutations have hinted at a critical role of LRRK2 in regulating dopamine release in the striatum ${ }^{[18,19]}$. However, as the results are highly inconsistent across studies, further efforts are needed to clarify the precise alterations induced by LRRK2 mutations. Here, by using gene-targeted knock-in (KI) mice, we systematically investigated the alterations in striatal electrochemical, electrophysiological, and motor function associated with LRRK2 RC and GS mutations.

35

\section{Methods (1627 words)}

\section{Mice}

All experiments were in compliance with Northwestern University Animal Care and Use Committee 40 guidelines. For electrophysiological studies, Drd1a-tdTomato mice (Jackson Laboratory 016204) were crossed with R1441C mice (Jackson Laboratory 009346) ${ }^{[20]}$ and Drd2-eGFP mice (MMRC 000230) were crossed with G2019S mice ${ }^{[21]}$. All mice were maintained on the C57BL/6 (Jax 000664) background. Heterozygotes for mutant LRRK2 alleles and their littermate controls, or wild-type (WT) mice, were used in all experiments. For electrophysiological recordings, hemizygotes for Drd1a-tdTomato and Drd2-eGFP were used for cellular 45 identification. Mice were group-housed on a standard 12/12 hr light/dark cycle. Both males and females were used in this study.

\section{Visualized ex vivo electrophysiology}

Mice at postnatal day 90-110 were anesthetized with a ketamine-xylazine mixture and perfused 50 transcardially with ice-cold aCSF containing the following (in mM): $125 \mathrm{NaCl}, 2.5 \mathrm{KCl}, 1.25 \mathrm{NaH}_{2} \mathrm{PO}_{4}, 2.0 \mathrm{CaCl}_{2}, 1.0$ $\mathrm{MgCl}_{2}, 25 \mathrm{NaHCO}_{3}$, and 12.5 glucose, bubbled continuously with carbogen $\left(95 \% \mathrm{O}_{2}\right.$ and $\left.5 \% \mathrm{CO}_{2}\right)$. The brains were rapidly removed, glued to the stage of a vibrating microtome (Leica Instrument), and immersed in ice-cold aCSF. 
Parasagittal slices containing the dorsal striatum were cut at a thickness of $240 \mu \mathrm{m}$ and transferred to a holding chamber where they were submerged in aCSF at $37^{\circ} \mathrm{C}$ for 30 min and maintained at room temperature thereafter.

55 Slices were then transferred to a small-volume $(\sim 0.5 \mathrm{ml})$ Delrin recording chamber mounted on a fixed-stage, upright microscope (Olympus). Neurons were visualized using differential interference contrast optics (Olympus), illuminated at $735 \mathrm{~nm}$ (Thorlabs), and imaged with a 60x water-immersion objective (Olympus) and a CCD camera (QImaging). Genetically-defined neurons were identified by somatic eGFP or tdTomato fluorescence examined under epifluorescence microscopy with a white (6,500 K) LED (Thorlabs) and appropriate filters (Semrock). glass (Sutter Instrument) pulled on a Flaming-Brown puller (Sutter Instrument) and fire-polished with a microforge (Narishige) immediately before use. Pipette resistance was typically $\sim 3-4 \mathrm{M} \Omega$. For whole-cell current-clamp recordings, the internal solution consisted of the following (in $\mathrm{mM}$ ): $135 \mathrm{KMeSO}_{4}, 10 \mathrm{Na}_{2}$ phosphocreatine, $5 \mathrm{KCl}, 5$ EGTA, 5 HEPES, $2 \mathrm{Mg}_{2} \mathrm{ATP}, 0.5 \mathrm{CaCl}_{2}$, and $0.5 \mathrm{Na}_{3} \mathrm{GTP}$, with $\mathrm{pH}$ adjusted to $7.25-7.30$ with $\mathrm{KOH}$. The liquid junction 65 potential for this internal solution was $\sim 7 \mathrm{mV}$ and was not corrected. For voltage-clamp recordings, neurons were clamped at $-80 \mathrm{mV}$ with an internal solution that contained the following (in $\mathrm{mM}$ ): $125 \mathrm{CsMeSO}_{3}, 10 \mathrm{Na}_{2}^{-}$

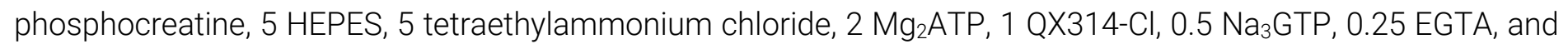
0.2\% (w/v) biocytin, with $\mathrm{pH}$ adjusted to 7.25-7.30 with $\mathrm{CsOH}$. Stimulus generation and data acquisition were performed using an amplifier (Molecular Devices), a digitizer (Molecular Devices), and pClamp (Molecular Devices). 70 For current-clamp recordings, the amplifier bridge circuit was adjusted to compensate for electrode resistance and was subsequently monitored. The signals were filtered at $1 \mathrm{kHz}$ and digitized at $10 \mathrm{kHz}$. $\mathrm{KMeSO}_{4}$ and $\mathrm{Na}_{2}-\mathrm{GTP}$ were from ICN Biomedicals and Roche, respectively. All other reagents were obtained from Sigma-Aldrich.

To determine the excitability of SPNs, the frequency-current (F-I) relationship of each cell was examined with current-clamp recordings. A series of current steps of 500 ms duration were applied beginning at -150 pA 75 and incremented at 25 pA for each consecutive sweep. This protocol was applied until each recorded cell reached maximal firing and after entering a depolarization block. Resting membrane potential was monitored for stability and cells that varied $20 \%$ from mean baseline were excluded from the analysis.

Corticostriatal responses were recorded in voltage-clamp as previously described ${ }^{[22]}$. Electrical stimulation was performed using parallel bipolar tungsten electrodes (FHC) placed in layer 5 of the cortex. Stimulus width and 
80 intensity were adjusted via a constant current stimulator (Digitimer), to evoke a first excitatory postsynaptic current (EPSC) with an amplitude of 200-400 pA in the presence of the GABA receptor antagonist SR95531 (10 $\mu \mathrm{M})$. Whole-cell access was monitored with a $-5 \mathrm{mV}$ pulse throughout the recording. Membrane capacitance (Cm) was determined off-line as $\mathrm{Cm}=\mathrm{Q}_{\mathrm{t}} * \mathrm{~V}_{\text {test, }}$ where Qt was calculated as the integral of the transient current elicited by $\bigvee_{\text {test, a }} 10 \mathrm{mV}$ voltage step ${ }^{[23]}$. The paired-pulse ratio (PPR) for a given cell was calculated by taking the average of 85 the ratios of the second EPSC amplitude to the first EPSC amplitude for each recording sweep. Data were excluded if the series resistance of the patch pipette differed by $>20 \%$ between the two recordings.

\section{Fast-scanning cyclic voltammetry}

Brain slices were prepared as described above in the electrophysiology section. Carbon fiber $(7 \mu \mathrm{m}$ 90 diameter) (Goodfellow) electrodes were fabricated with glass capillary (Sutter) using a puller (Narishige) and fiber tips were hand-cut to 30-100 $\mu \mathrm{m}$ past the capillary tip. The carbon-fiber electrode was held at $-0.4 \mathrm{~V}$ before each scan. A voltage ramp to and from $1.2 \mathrm{~V}(400 \mathrm{~V} / \mathrm{s})$ was delivered every $100 \mathrm{~ms}(10 \mathrm{~Hz})$. Before recording, electrodes were conditioned by running the ramp at $60 \mathrm{~Hz}$ for $15 \mathrm{~min}$ and at $10 \mathrm{~Hz}$ for another 15 min and calibrated using 1 $\mu \mathrm{M}$ dopamine hydrochloride (Sigma). Dopamine transients were evoked by electrical stimulation delivered through

95 a concentric, bipolar electrode (FHC) placed in the rostrodorsal striatum (Figure 1b) because of its known and important involvement in reward and motivation ${ }^{[24-27]}$ and motor learning and control ${ }^{[27-29]}$. A single electrical pulse

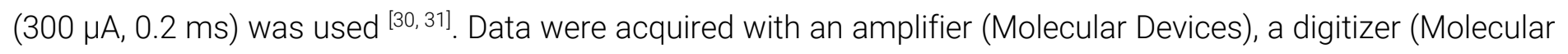
Devices), and pClamp (Molecular Devices). For each slice, four measurements were made and then averaged. The custom analysis was written in MATLAB (MathWorks). The voltammogram and peak oxidative current amplitudes 100 of the dopamine transient were measured. Experiments were rejected when the evoked current did not have the characteristic electrochemical signature of dopamine.

\section{Behavioral tests}

Motor learning was assessed with an accelerating rotarod in WT, RC, and GS mice. The task started when 105 the mice were around postnatal day 60 using a rotarod apparatus (Panlab) equipped with a mouse rod (3 cm 
diameter) and set to 4-40 rpm acceleration over $300 \mathrm{~s}$. The task consisted of eighteen daily sessions (five trials per session; intertrial-interval = 15 s, max trial duration = 300 s) divided into two phases ${ }^{[32]}$ (Figure 3a). Specifically, during the dopamine receptor antagonism phase (session 1-5), mice were systemically injected (i.p.) 30 min prior to testing with one of the following: $0.9 \%$ saline, cocktail of $1 \mathrm{mg} / \mathrm{kg} \mathrm{SCH} 23390+1 \mathrm{mg} / \mathrm{kg}$ eticlopride, $1 \mathrm{mg} / \mathrm{kg}$

110 SCH23390 or 1 mg/kg eticlopride. Following a 72-hr break, mice were then tested for another thirteen sessions (drug-free recovery phase). To determine if LRRK2 mutation contributes to motor learning deficit, a cohort of RC mice was administered with $5 \mathrm{mg} / \mathrm{kg}$ MLi-2 (LRRK2 inhibitor) ${ }^{[33,34]} 60$ min prior to all daily sessions and saline or cocktail of $1 \mathrm{mg} / \mathrm{kg} \mathrm{SCH} 23390+1 \mathrm{mg} / \mathrm{kg}$ eticlopride was given $30 \mathrm{~min}$ before the first five daily sessions. All the injections were given i.p $(0.1 \mathrm{ml} / \mathrm{kg})$. A separate cohort of WT, RC, and GS mice underwent the accelerating rotarod

115 task for the initial drug-treated phase. Before the initiation of the drug-free recovery phase (after the 72-hr break), mice were assessed individually in 56 × $56 \mathrm{~cm}$ open-field arenas in noise-canceling boxes and illuminated by dim red lights. Session (five-minute duration) started when mice were placed in the center of the arena. Locomotor activity was analyzed by the LimeLight (Actimetrics) software and reported as distance traveled.

\section{Western blot analysis}

Mice were treated with either MLi-2 (5 mg/kg, i.p.) or vehicle for $60 \mathrm{~min}$; the striata were dissected and rapidly homogenized in four volumes of ice-cold Buffer $\mathrm{A}(0.32 \mathrm{M}$ sucrose, $5 \mathrm{mM}$ HEPES, $1 \mathrm{mM} \mathrm{MgCl}$, $0.5 \mathrm{mM}$ $\mathrm{CaCl}_{2}, \mathrm{pH}$ 7.4) supplemented with Halt protease and phosphatase inhibitor cocktail (Thermo Fisher Scientific) using a Teflon homogenizer (12 strokes). Homogenized striatal extract was centrifuged at 1,400 g for $10 \mathrm{~min} .20$ $125 \mu \mathrm{g}$ of the supernatant were separated by 4-12\% NuPage Bis-Tris PAGE (Thermo Fisher Scientific) and transferred to membranes using the iBlot nitrocellulose membrane Blotting system (Thermo Fisher Scientific) by following manufacturer's protocol. Primary antibodies specific for pS935 LRRK2 (Abcam, ab230261 1:1000), total LRRK2 (Abcam, ab133476), and $\beta$-actin (Thermo Fisher Scientific, MA1-045 1:3000) were used. Secondary anti-mouse and anti-rabbit antibodies were from Thermo Fisher Scientific Membranes were incubated with Immobilon ECL 130 Ultra Western HRP Substrate (Millipore) for 3 min prior to image acquisition. Chemiluminescent blots were imaged with iBright CL1000 imaging system (Thermo Fisher Scientific). 


\section{Experimental design and statistical analyses}

General graphing and statistical analyses were performed with MATLAB (MathWorks), SAS (SAS Institute), 135 and Prism (GraphPad). Custom analysis codes are available on GitHub (https://github.com/chanlab). Sample size (n value) is defined by the number of observations (i.e., neurons or cells). No statistical method was used to predetermine the sample size. Unless noted otherwise, data are presented as median values \pm median absolute deviations as measures of central tendency and statistical dispersion, respectively. Box plots are used for graphic representation of population data ${ }^{[35,36]}$. The central line represents the median, the box edges represent the 140 interquartile ranges, and the whiskers represent $10-90^{\text {th }}$ percentiles. Normal distributions of data were not assumed for electrophysiological data. Comparisons for unrelated samples were performed using Mann-Whitney $U$ test at a significance level (a) of 0.05 . Unless noted otherwise, exact $\mathrm{P}$ values are reported. Behavioral data are presented as mean \pm standard error of the mean and were analyzed with either two-way or three-way ANOVA with repeated measures followed by Tukey post hoc tests.

Results (2169 words)

\section{R1441C and G2019S LRRK2 mice have decreased nigrostriatal dopaminergic transmission}

Studies that examine dopamine transmission in LRRK2 mutant mice have not yielded consistent results $150^{[18,37-40]}$. This was in part due to the employment of bacterial artificial chromosomes (BAC) or other transgenic lines confounded by unintended genomic alterations in expression patterns and levels of endogenous LRRK2 ${ }^{[41,42]}$. KI mouse models serve as a well-validated approach for studying LRRK2 mutations in relevant cell types at the physiologically-relevant expression level. In this study, we used adult LRRK2 RC and GS KI mice that express mutant LRRK2 proteins under the regulation of the endogenous promoter. We first examined nigrostriatal 155 dopamine transmission with fast-scanning cyclic voltammetry in ex vivo striatal tissues from WT, RC, and GS mice

(Figure 1b). Here, we found a decrease in evoked dopamine release in RC mice compared to WT ([DA $]_{W T}=856.5$ $\pm 198.4 \mathrm{nM}, \mathrm{n}=13$ recordings; $[\mathrm{DA}]_{\mathrm{RC}}=516.7 \pm 148.6 \mathrm{nM}, \mathrm{n}=19$ recordings; $p=0.0024$, Mann-Whitney $U$ test $)$. In addition, we found a similar decrease in evoked dopamine release in GS mice compared to WT ([DA $]_{\text {GS }}=562.4 \pm$ 
$228.3 \mathrm{nM}, \mathrm{n}=19$ recordings; $p=0.0030$, Mann-Whitney $U$ test). There were no differences in evoked dopamine 160 release between RC and GS mice ( $p=0.89$, Mann-Whitney $U$ test) (Figure 1c \& d).

\section{iSPNs have decreased excitability in R1441C LRRK2 mice}

It is established that dopamine modulates the excitability of striatal projection neurons in naive animals ${ }^{[43-}$ ${ }^{47]}$. Accordingly, the disruption of dopamine signaling profoundly alters the intrinsic properties of SPNs ${ }^{[22,48]}$. Given 165 our finding that both RC and GS mice had decreased dopamine release, we sought to examine if direct- and indirect-pathway striatal projection neurons (dSPNs and iSPNs) have altered excitability in the LRRK2 mutant mice. Whole-cell, current-clamp recordings were performed on identified SPNs in WT, RC, and GS mice (Figure 2).

We found no differences in the number of spikes in dSPNs between WT and RC mice for the current (I) that elicited the half-maximum firing from dPSNs in the WT mice $(I=475$ pA, outputwT $=12.0 \pm 4.0$ spikes, $n=20$

170 recordings; output $_{\mathrm{RC}}=14.0 \pm 3.0$ spikes, $\mathrm{n}=30$ recordings; $p=0.21$, Mann - Whitney $U$ test $)$ (Figure 2a). In contrast, there was a decrease in the number of evoked spikes for the iSPNs in RC mice for the current that yielded the half-

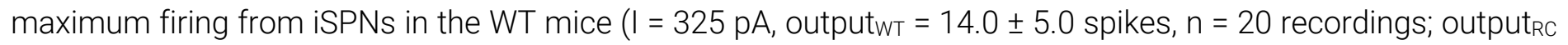
$=6.0 \pm 6.0$ spikes, $n=23$ recordings; $p=0.037$; Mann-Whitney $U$ test) (Figure $\mathbf{2 b}$ ). To quantify the decreased excitability in the iSPNs of RC mice, we compared input-output functions for the WT and RC mice. As shown in

175 Figure $\mathbf{2} \mathbf{b}$, the maximal difference in the spike output was observed at I = 625 pA; a lower number of spikes were elicited in iSPNs of RC mice compared to WT mice (output ${ }_{W T}=22.0 \pm 4.5$ spikes, $n=17$ recordings; output $_{\mathrm{RC}}=15.0$ \pm 5.0 spikes, $n=23$ recordings; $p=0.0010$, Mann-Whitney $U$ test) (Figure $\mathbf{2 b}$ ). In contrast, we found no detectable differences in GS mice as measured with the half-maximum firing responses for either dSPNs (outputwT $=8.0 \pm$ 5.0 spikes, $n=13$ recordings; output ${ }_{\mathrm{GS}}=11.0 \pm 3.0$ spikes, $\mathrm{n}=29$ recordings; $p=0.12$, Mann-Whitney $U$ test $)$ or

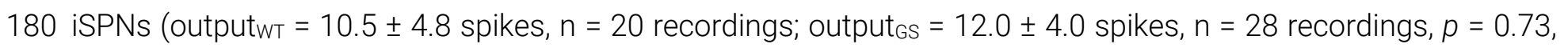
Mann-Whitney $U$ test) (Figure 2c \& d).

As the excitability of SPNs is a function of dendritic structure ${ }^{[49]}$, we asked whether an increase in membrane capacitance $(\mathrm{Cm})$ could account for the decreased excitability seen in the iSPNs of RC mice. We found an increased membrane capacitance for iSPNs in RC mice compared to WT mice $\left(\mathrm{Cm}_{W T}=68.8 \pm 13.9 \mathrm{pF}, \mathrm{n}=27\right.$; 
$185 \mathrm{Cm}_{\mathrm{RC}}=85.2 \pm 13.3 \mathrm{pF}, \mathrm{n}=27 ; \mathrm{p}=0.039$, Mann-Whitney $U$ Test). In contrast, no change in membrane capacitance was observed for dSPNs between RC and WT mice $\left(\mathrm{Cm}_{\mathrm{WT}}=79.4 \pm 19.5 \mathrm{pF}, \mathrm{n}=25\right.$ recordings; $\mathrm{Cm}_{\mathrm{RC}}=85.1 \pm 16.0$ $\mathrm{pF}, \mathrm{n}=38$ recordings; $p=0.98$, Mann-Whitney $\cup$ Test) (Figure 2-figure supplement 1). We also examined for changes in capacitance in GS mice. In line with previous findings [50,51], we found no changes in the intrinsic properties of SPNs in the GS mice. We found no differences in membrane capacitance between GS mutant or WT 190 mice for dSPNs $\left(\mathrm{Cm}_{\mathrm{WT}}=88.1 \pm 10.8 \mathrm{pF}, \mathrm{n}=15\right.$ recordings; $\mathrm{Cm}_{\mathrm{GS}}=95.4 \pm 12.0 \mathrm{pF}, \mathrm{n}=27$ recordings; $p=0.29$, Mann-Whitney U Test) or iSPNs (CmWT $=74.6 \pm 12.3 \mathrm{pF}, \mathrm{n}=19$ recordings; $\mathrm{Cm}_{\mathrm{GS}}=81.8 \pm 15.2 \mathrm{pF}, \mathrm{n}=30$ recordings; $p=0.51$, Mann-Whitney U Test) (Figure 2-figure supplement 1).

The hypoexcitability of iSPNs in models of PD has been suggested as a homeostatic response to an increased corticostriatal transmission ${ }^{[50,52,53]}$, we thus examined possible presynaptic changes in corticostriatal 195 transmission by measuring the paired-pulse ratios (PPRs) of the corticostriatal responses in WT and RC mice (see Methods). There were no differences in the PPRs for either dSPNs (PPRWT $=1.31 \pm 0.10, n=14$ recordings; PPRRC $=1.33 \pm 0.15, n=17$ recordings; $p=0.18$, Mann-Whitney $U$ test) or iSPNs (PPRWT $=1.28 \pm 0.14, n=13$ recordings; $\mathrm{PPR}_{\mathrm{RC}}=1.54 \pm 0.25, \mathrm{n}=18$ recordings; $p=0.11$, Mann-Whitney $U$ test) (Figure 2-figure supplement 2) Unexpectedly, we found an increase in the 95\%-5\% EPSC decay time of iSPNs in RC mice compared to WT mice 200 (decay $_{w T}=61.1 \pm 38.7 \mathrm{~ms}, \mathrm{n}=13$ recordings; decay $_{\mathrm{RC}}=139.5 \pm 81.1 \mathrm{~ms}, \mathrm{n}=18$ recordings; $p=0.037$, MannWhitney $U$ test) (Figure 2-figure supplement 2). In contrast, there was no difference in EPSC decay times for dSPNs between WT mice and RC mice (decay $\mathrm{wT}_{\mathrm{T}}=88.7 \pm 40.3 \mathrm{~ms}, \mathrm{n}=14$ recordings; decay $_{\mathrm{RC}}=182.9 \pm 136.0 \mathrm{~ms}$ $\mathrm{n}=17$ recordings; $p=0.13$, Mann-Whitney $U$ test) (Figure 2-figure supplement 2).

\section{R1441C mice show impaired dopamine-dependent motor learning}

The dorsal striatum plays a critical role in habit and motor learning ${ }^{[54-57]}$. Given the reduction of dopamine release in the dorsal striatum in RC and GS KI mice (Figure 1) we sought to investigate how a disruption to dopamine signaling would affect motor learning. Specifically, we first systemically injected D1 and D2 dopamine receptor antagonists (SCHSCH23390 and eticlopride, referred to as "antagonists cocktail") to determine the roles 
210 of D1 and D2 dopamine receptor-mediated signaling on motor learning in RC and GS mice. We then used an accelerating rotarod task-a well-established paradigm for assessing striatally dependent motor learning ${ }^{[32,58-60]}$.

Consistent with previous studies that RC KI mice display no overt abnormalities in striatum-dependent motor learning under basal conditions ${ }^{[20,61]}$, we found that saline treated RC mice learned equally well on the rotarod task (Figure $\mathbf{3 b}$ ). Our data consistent with prior reports in WT mice ${ }^{[32,58]}$ showed that mice treated with 215 antagonists cocktail during the initial five-day training period (session 1-5; Figure 3a) have dramatic impairments in the rotarod performance, regardless of genotype.

Here, we showed that upon 72 hrs of recovery from the last antagonist administration, the latency to fall for WT, RC, and GS mice although initially degraded (compared to saline treated controls), (Figure 3b), increased over subsequent sessions in different manners (saline: $\mathrm{n}_{W T}=10$ mice, $\mathrm{n}_{\mathrm{RC}}=10$ mice, and $\mathrm{n}_{\mathrm{GS}}=9$ mice; $\mathrm{D} 1+\mathrm{D} 2$ 220 receptor antagonists: $\mathrm{n}_{\mathrm{WT}}=13$ mice, $\mathrm{n}_{\mathrm{RC}}=11$ mice, and $\mathrm{n}_{\mathrm{GS}}=11$ mice; treatment $\mathrm{x}$ genotype $\mathrm{x}$ session interaction, $F_{102,1887}=1.95, p<0.001$, 3-way ANOVA with repeated measures) (Figure $\mathbf{3 b}$ ). The average latency to fall in the drug-treated WT and GS mice showed no difference compared to saline-treated controls over the training sessions

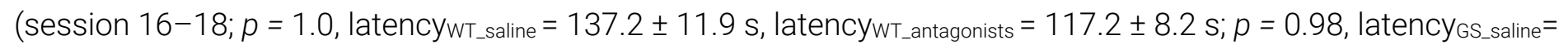
$130.9 \pm 8.7$ s, latency ${ }_{G S \_a n t a g o n i s t s}=99.2 \pm 15.6$ s, 3-way ANOVA, repeated measures) (Figure 3b \& c, Figure 3-table

225 supplement 1). In contrast, dopamine receptor antagonism unmasked a deficit in RC KI mice; they failed to reach the performance of their corresponding saline-treated controls over the training sessions (trial x genotype $x$ drug interaction: $F_{6,116}=2.24, p=0.044$; latency ${ }_{\mathrm{RC} \_s a l i n e}=162.4 \pm 17.0 \mathrm{~s}$, latency $\mathrm{RC}_{\mathrm{R} \_ \text {antagonists }}=58.5 \pm 11.1 \mathrm{~s}, p<0.001,3-$ way ANOVA with Tukey post hoc test) (Figure 3c). Similarly, RC KI mice failed to reach the performance of their dopamine D1 and D2 receptor antagonist treated WT controls (trial $x$ genotype $x$ drug interaction: $F_{6,116}=2.24, p=$

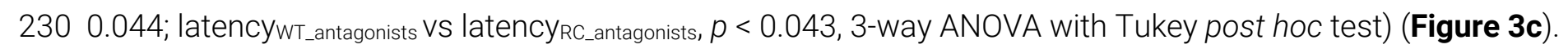

Given that LRRK2 mutations stimulate LRRK2 kinase activity, we examined whether increased kinase activity in RC mice contributes to this motor learning deficit. Therefore, we treated RC mice with the LRRK2 inhibitor MLi-2 ${ }^{[33]}$ prior to D1 and D2 receptor antagonism. We found motor improvement across behavioral sessions during the drug-free phase of MLi-2-treated mice; this was evident with an increased latency to fall across 235 successive trials (time $x$ treatment interaction, $F_{17,374}=12.45, p<0.001$, two-way ANOVA) (Figure 3d). This 
inference was confirmed by analyzing the average latency in mice with and without MLi-2 treatment. Increase in latency was only observed following MLi-2 injections (time $x$ treatment interaction, $\mathrm{F}_{3,60}=10.98, p<0.001$; for $\mathrm{MLi}$ 2 with D1+D2 antagonism: $p=0.002$, latency session_7-9 $_{2}=38.7 \pm 7.0 \mathrm{~s}$, latency session_10-12 $_{2}=67.7 \pm 6.4 \mathrm{~s} ; p=0.001$, vs latency $_{\text {session_13-15 }}=90.6 \pm 7.9 \mathrm{~s} ; p<0.001$, vs latency session_16-18 $_{\text {s }}=112.8 \pm 11.0$ s, two-way ANOVA with Tukey post 240 hoc tests) (Figure 3e). The pre-treatment of MLi-2 decreased LRRK2 S935 phosphorylation-a well-characterized

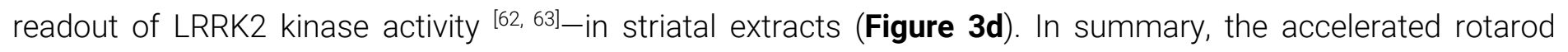
paradigm with dopamine antagonist treatment unmasked the kinase-mediated deficits of striatal motor learning in $\mathrm{RC}$ mice.

To rule out the possibility that the impaired learning of RC mice was a lingering effect of the antagonist 245 treatment, a subgroup of RC mice that received the administration of dopamine receptor antagonists were returned to their home cages without rotarod training (here referred to as "untrained mice"). After the 72-hour break, these mice were tested on the rotarod task; their performance was improved compared to the RC mice that underwent training in the rotarod task immediately following systemic administration of dopamine receptor antagonists, as measured by latency to fall (main effect of treatment, $F_{1,260}=189.2, p<0.0001$, two-way ANOVA)

\section{0 (Figure 3-figure supplement 1).}

To examine the contributions of dopamine receptor subtype in shaping motor learning on the rotarod task, we administered D1 and D2 receptor antagonists separately during the initial acquisition. The administration of $\mathrm{SCH} 23390$ or eticlopride impaired the performance during the initial phase of the rotarod training across all genotypes (session 1-5), compared to their saline-treated controls (Figure 3-figure supplement 1. While D1 255 receptor antagonism led to an immediate improvement in all genotypes in the drug-free phase (Figure 3-figure supplement 1), D2 receptor antagonism resulted in a delayed improvement in performance (Figure 3-figure supplement 1; Figure 3-table supplement 1). In addition, WT and GS mice treated with D2 receptor antagonist reached the performance of their saline controls by the end of the task (latency GS_eticlopride $=105.9 \pm 9.2 \mathrm{~s}$,

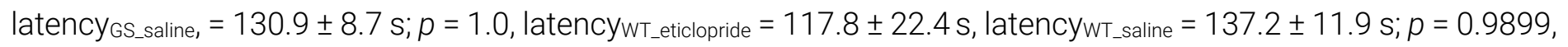
260 3-way ANOVA, Tukey post hoc test). In contrast, RC mice did not exhibit the performance of their saline controls 
across sessions (latency $\mathrm{RC}_{\mathrm{R}}$ eticlopride $=106.3 \pm 12.7 \mathrm{~s}$, latency $\mathrm{RC}_{\mathrm{R} \text { saline, }}=162.4 \pm 17.0 \mathrm{~s} p=0.016$; 3-way ANOVA with Tukey post hoc test).

To show that the motor learning impairment in RC mice were not confounded by locomotor deficits, we examined the locomotor behavior of mice in the open field arena. WT, RC, and GS mice that underwent dopamine 265 antagonism and an initial five-day rotarod training were assessed by the total distance traveled. We found no differences between any of the groups except for a significant main effect of drug treatment (distancewT_saline $=$ $28.48 \pm 3.36 \mathrm{~m}$; distance $W_{\text {T_antagonists }}=24.44 \pm 2.90 \mathrm{~m}$; distance $\mathrm{RC} \_$saline $=36.02 \pm 2.26 \mathrm{~m}$; distance $\mathrm{RC} \_$antagonists $=30.62$ $\pm 4.48 \mathrm{~m}$; distance GS_saline $_{2}=31.91 \pm 2.19 \mathrm{~m}$; distance di_antagonists $_{2}=30.85 \pm 3.49 \mathrm{~m}$; treatment effect, $F_{3,83}=4.63, p=$ 0.0048; genotype effect, $F_{2,83}=0.61, p=0.54$; treatment $x$ genotype effect, $F_{6,83}=1.94, p=0.084$, 3-way ANOVA)

270 (Figure 3-figure supplement 1). In summary, disruption of dopamine signaling during rotatord training task specifically interferes with striatal motor learning in the RC mice, which otherwise exhibit no deficits in naturalistic behavior such as locomotion.

\section{Discussion (851 words)}

While a number of transgenic animal models have been generated to interrogate dysfunction associated with mutant LRRK2, the findings so far have been inconsistent. The most parsimonious explanation is the difference in expression levels of mutant LRRK2 in the presence of endogenous LRRK2, as well as the different promoters used to drive the expression of mutant protein ${ }^{[18]}$. In this study, by using RC and GS KI mice that express 280 the mutant LRRK2 protein with endogenous expression patterns and levels, we performed a systematic comparative study between these two Lrrk2 KI mouse models to resolve conflicting reports of dopamine transmission ${ }^{[21,51]}$. We found a decrease in nigrostriatal dopamine release in both RC and GS mice. Additionally, iSPNs in the RC mice showed a selective decrease in excitability, while no differences were found in dSPNs. The alterations in the cellular properties of iSPNs in RC mice were paralleled with impairments in dopamine-dependent 285 striatal motor learning. We currently do not fully understand the dissociation between impaired dopamine release and striatal alterations. We speculate that PKA signaling at the striatal level may be dysregulated due to RC 
mutation ${ }^{[64,65]}$. This is in line with our finding that increased synaptic PKA activities are observed only in RC and not GS striatal synaptic fractions ${ }^{[15,66]}$

To our knowledge, this study is the first to examine evoked dopamine release in the RC KI mice. Our results 290 are at odds with an earlier report that measured basal dopamine content in RC KI mice and found no changes using bulk tissue HPLC, which lacks the spatial and temporal resolution that fast-scanning cyclic voltammetry ${ }_{\text {offers }}{ }^{[20]}$. On the other hand, our data corroborate the finding that stimulated catecholamine release in cultured chromaffin cells of RC mice had a 50\% reduction of dopamine release, ${ }^{[20]}$. The decrease of evoked dopamine release could be attributed to the role of LRRK2 in regulating presynaptic vesicle release ${ }^{[67-70]}$. In particular, LRRK2 295 targets downstream Rab proteins that are involved in vesicular trafficking to the plasma membrane and direct neurotransmitter release ${ }^{[12,71]}$. Specifically, we recently demonstrated that RC mutation resulted in higher phosphorylation of Rab8A-a downstream LRRK2 substrate-in synaptic striatal extracts, compared to GS mutation ${ }^{[15]}$. In addition, the LRRK2 RC mutation leads to a synaptic translocation and dysregulation of excitatory

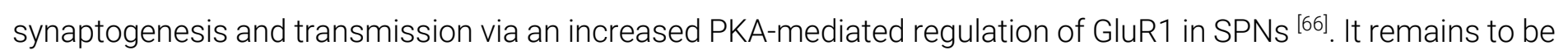
300 determined if these alterations are specific to iSPNs and if they are associated with the hypoexcitability observed in our study. Overall, our results show that the RC mutation has a more profound effect on striatal physiology.

In the present study we showed that GS mutation caused decreased evoked dopamine release. Previous studies that measured dopamine in GS KI mice have been contradictory with one another. Using microdialysis, reduced extracellular levels of dopamine at twelve months but not six months were reported ${ }^{[21]}$. In contrast, by 305 using amperometry Tozzi et al. reported reduced striatal dopamine levels in mice at six months ${ }^{[51]}$. Moreover, a recent report showed no difference on peak dopamine dopamine release in slices of three months ${ }^{[50]}$. However, when $\sim 70 \%$ maximum dopamine release was evoked by a single stimuli, they reported a significant increase in dopamine release in GS mice. There are several possibilities for the variability across the results including age of animals, genetic backgrounds, methods to evaluate dopamine content, and heterogeneity across striatal 310 subregions of dopamine release in different experiments.

Given the importance of nigrostriatal dopamine signaling in striatal motor learning, we assessed motor learning in both RC and GS KI lines using the accelerated rotarod. Consistent with previous studies, we observed no abnormalities in motor learning in LRRK2 mutants under basal conditions ${ }^{[20,61]}$. While our data are in agreement 
with previous studies showing that WT mice that underwent dopamine receptor antagonist treatment have an

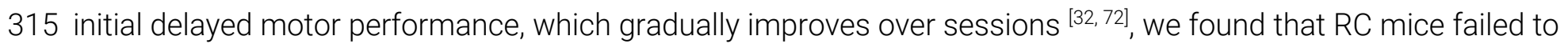
improve their performance with time. This was not the case for the GS mice. Notably, our findings argue that increased LRRK2 kinase activity underlies this impairment as pretreatment of the mice with the LRRK2 kinase inhibitor improved the performance of RC mice throughout sessions. It is not clear at this point why RC and not GS mice demonstrate this LRRK2 kinase-dependent motor impairment but this finding is in alignment with 320 emerging data demonstrating that mutations outside of the kinase domain lead to greater increase in kinase activity than those found in the kinase domain itself ${ }^{[1]}$.

Previously, it was suggested that the combination of dopamine antagonists and rotarod training leads to

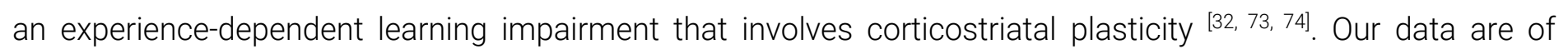
particular importance as they mirror subclinical dopaminergic dysfunction and corticostriatal alterations of the 325 asymptomatic LRRK2 mutation carriers [75-77]; our study argues that the behavioral deficit associated with LRRK2 mutation is reversed by LRRK2 targeted therapeutic innervations.

Overall, the investigation of the preclinical symptoms of dopamine and striatal dysfunctions in the mutant LRRK2 KI mice provides the framework for implementing neuroprotective therapies and developing biomarkers to detect and monitor disease progression related to LRRK2 mutations. Specifically, given the striatal alterations we 330 observed were in the RC mutant mice, our study highlights the importance of future studies focused on the mutations of the GTPase domain and its downstream signaling targets for the development of signaling-specific neuroprotective therapies.

\section{References}

335 1. Alessi, D.R. and E. Sammler, LRRK2 kinase in Parkinson's disease. Science, 2018. 360(6384): p. 36-37.

2. Cookson, M.R., The role of leucine-rich repeat kinase 2 (LRRK2) in Parkinson's disease. Nat Rev Neurosci, 2010. 11(12): p. 791-7.

3. Satake, W., et al., Genome-wide association study identifies common variants at four loci as genetic risk factors for Parkinson's disease. Nat Genet, 2009. 41(12): p. 1303-7. 
340 4. Nalls, M.A., et al., Large-scale meta-analysis of genome-wide association data identifies six new risk loci for Parkinson's disease. Nat Genet, 2014. 46(9): p. 989-93.

5. Simon-Sanchez, J., et al., Genome-wide association study reveals genetic risk underlying Parkinson's disease. Nat Genet, 2009. 41(12): p. 1308-12.

6. Di Fonzo, A., et al., Comprehensive analysis of the LRRK2 gene in sixty families with Parkinson's disease. $345 \quad$ Eur J Hum Genet, 2006. 14(3): p. 322-31.

7. Haugarvoll, K., et al., Lrrk2 R1441C parkinsonism is clinically similar to sporadic Parkinson disease. Neurology, 2008. 70(16 Pt 2): p. 1456-60.

8. Kluss, J.H., A. Mamais, and M.R. Cookson, LRRK2 links genetic and sporadic Parkinson's disease. Biochem Soc Trans, 2019. 47(2): p. 651-661.

350 9. Paisan-Ruiz, C., P.A. Lewis, and A.B. Singleton, LRRK2: cause, risk, and mechanism. J Parkinsons Dis, 2013. 3(2): p. 85-103.

10. Dauer, W. and C.C. Ho, The biology and pathology of the familial Parkinson's disease protein LRRK2. Mov Disord, 2010. 25 Suppl 1: p. S40-3.

11. Esteves, A.R., R.H. Swerdlow, and S.M. Cardoso, LRRK2, a puzzling protein: insights into Parkinson's 355 disease pathogenesis. Exp Neurol, 2014. 261: p. 206-16.

12. Steger, M., et al., Phosphoproteomics reveals that Parkinson's disease kinase LRRK2 regulates a subset of Rab GTPases. Elife, 2016. 5.

13. Liu, Z., et al., LRRK2 phosphorylates membrane-bound Rabs and is activated by GTP-bound Rab7L1 to promote recruitment to the trans-Golgi network. Hum Mol Genet, 2018. 27(2): p. 385-395.

360 14. Purlyte, E., et al., Rab29 activation of the Parkinson's disease-associated LRRK2 kinase. EMBO J, 2018. 37(1): p. 1-18.

15. Chen, C., et al., Pathway-specific deregulation of striatal excitatory synapses in LRRK2 mutations. bioRxiv, 2020: p. 2020.01.03.894410.

16. Shu, L., et al., Clinical Heterogeneity Among LRRK2 Variants in Parkinson's Disease: A Meta-Analysis. Front Aging Neurosci, 2018. 10: p. 283. 
17. Dubbioso, R., et al., Does motor cortex plasticity depend on the type of mutation in the leucine-rich repeat kinase 2 gene? Mov Disord, 2017. 32(6): p. 947-948.

18. Volta, M. and H. Melrose, LRRK2 mouse models: dissecting the behavior, striatal neurochemistry and neurophysiology of PD pathogenesis. Biochem Soc Trans, 2017. 45(1): p. 113-122.

370 19. Xiong, Y., T.M. Dawson, and V.L. Dawson, Models of LRRK2-Associated Parkinson's Disease. Adv Neurobiol, 2017. 14: p. 163-191.

20. Tong, Y., et al., R1441C mutation in LRRK2 impairs dopaminergic neurotransmission in mice. Proc Natl Acad Sci U S A, 2009. 106(34): p. 14622-7.

21. Yue, M., et al., Progressive dopaminergic alterations and mitochondrial abnormalities in LRRK2 G2019S knock-in mice. Neurobiol Dis, 2015. 78: p. 172-95.

22. Chan, C.S., et al., Strain-specific regulation of striatal phenotype in Drd2-eGFP BAC transgenic mice. J Neurosci, 2012. 32(27): p. 9124-32.

23. Hernandez, V.M., et al., Parvalbumin+ Neurons and Npas1+ Neurons Are Distinct Neuron Classes in the Mouse External Globus Pallidus. J Neurosci, 2015. 35(34): p. 11830-47.

380 24. Haber, S.N., et al., Reward-related cortical inputs define a large striatal region in primates that interface with associative cortical connections, providing a substrate for incentive-based learning. J Neurosci, 2006. 26(32): p. 8368-76.

25. Elliott, R., et al., Differential response patterns in the striatum and orbitofrontal cortex to financial reward in humans: a parametric functional magnetic resonance imaging study. J Neurosci, 2003. 23(1): p. 303-7.

385 26. Schultz, W., L. Tremblay, and J.R. Hollerman, Reward processing in primate orbitofrontal cortex and basal ganglia. Cereb Cortex, 2000. 10(3): p. 272-84.

27. Corlett, P.R., et al., Prediction error during retrospective revaluation of causal associations in humans: fMRI evidence in favor of an associative model of learning. Neuron, 2004. 44(5): p. 877-88.

28. Wickens, J.R., J.N. Reynolds, and B.I. Hyland, Neural mechanisms of reward-related motor learning. Curr Opin Neurobiol, 2003. 13(6): p. 685-90.

29. McFarland, N.R. and S.N. Haber, Convergent inputs from thalamic motor nuclei and frontal cortical areas to the dorsal striatum in the primate. J Neurosci, 2000. 20(10): p. 3798-813. 
30. Lemos, J.C., et al., Enhanced GABA Transmission Drives Bradykinesia Following Loss of Dopamine D2 Receptor Signaling. Neuron, 2016. 90(4): p. 824-38.

395 31. Adrover, M.F., J.H. Shin, and V.A. Alvarez, Glutamate and dopamine transmission from midbrain dopamine neurons share similar release properties but are differentially affected by cocaine. J Neurosci, 2014. 34(9): p. 3183-92.

32. Beeler, J.A., et al., A role for dopamine-mediated learning in the pathophysiology and treatment of Parkinson's disease. Cell Rep, 2012. 2(6): p. 1747-61.

400 33. Fell, M.J., et al., MLi-2, a Potent, Selective, and Centrally Active Compound for Exploring the Therapeutic Potential and Safety of LRRK2 Kinase Inhibition. J Pharmacol Exp Ther, 2015. 355(3): p. 397-409.

34. Scott, J.D., et al., Discovery of a 3-(4-Pyrimidinyl) Indazole (MLi-2), an Orally Available and Selective LeucineRich Repeat Kinase 2 (LRRK2) Inhibitor that Reduces Brain Kinase Activity. J Med Chem, 2017. 60(7): p. 2983-2992.

405 35. Krzywinski, M. and N. Altman, Visualizing samples with box plots. Nat Methods, 2014. 11(2): p. 119-20.

36. Streit, M. and N. Gehlenborg, Bar charts and box plots. Nat Methods, 2014. 11(2): p. 117.

37. Li, X., et al., Enhanced striatal dopamine transmission and motor performance with LRRK2 overexpression in mice is eliminated by familial Parkinson's disease mutation G2019S. J Neurosci, 2010. 30(5): p. 178897.

410 38. Li, Y., et al., Mutant LRRK2(R1441G) BAC transgenic mice recapitulate cardinal features of Parkinson's disease. Nat Neurosci, 2009. 12(7): p. 826-8.

39. Ramonet, D., et al., Dopaminergic neuronal loss, reduced neurite complexity and autophagic abnormalities in transgenic mice expressing G2019S mutant LRRK2. PLoS One, 2011. 6(4): p. e18568.

40. Melrose, H.L., et al., Impaired dopaminergic neurotransmission and microtubule-associated protein tau 415 alterations in human LRRK2 transgenic mice. Neurobiol Dis, 2010. 40(3): p. 503-17.

41. Beil, J., et al., Is BAC transgenesis obsolete? State of the art in the era of designer nucleases. J Biomed Biotechnol, 2012. 2012: p. 308414.

42. Chaturvedi, P., et al., Stable and reproducible transgene expression independent of proliferative or differentiated state using BAC TG-EMBED. Gene Ther, 2018. 25(5): p. 376-391. 
420 43. Lahiri, A.K. and M.D. Bevan, Dopaminergic Transmission Rapidly and Persistently Enhances Excitability of D1 Receptor-Expressing Striatal Projection Neurons. Neuron, 2020.

44. Gerfen, C.R. and D.J. Surmeier, Modulation of striatal projection systems by dopamine. Annu Rev Neurosci, 2011. 34: p. 441-66.

45. Bernard, V., E. Normand, and B. Bloch, Phenotypical characterization of the rat striatal neurons expressing 425 muscarinic receptor genes. J Neurosci, 1992. 12(9): p. 3591-600.

46. Ericsson, J., et al., Dopamine differentially modulates the excitability of striatal neurons of the direct and indirect pathways in lamprey. J Neurosci, 2013. 33(18): p. 8045-54.

47. Ding, L. and D.J. Perkel, Dopamine modulates excitability of spiny neurons in the avian basal ganglia. J Neurosci, 2002. 22(12): p. 5210-8.

430 48. Fieblinger, T., et al., Cell type-specific plasticity of striatal projection neurons in parkinsonism and L-DOPAinduced dyskinesia. Nat Commun, 2014. 5: p. 5316.

49. Gertler, T.S., C.S. Chan, and D.J. Surmeier, Dichotomous anatomical properties of adult striatal medium spiny neurons. J Neurosci, 2008. 28(43): p. 10814-24.

50. Volta, M., et al., Initial elevations in glutamate and dopamine neurotransmission decline with age, as does 435 exploratory behavior, in LRRK2 G2019S knock-in mice. Elife, 2017. 6.

51. Tozzi, A., et al., Dopamine D2 receptor-mediated neuroprotection in a G2019S Lrrk2 genetic model of Parkinson's disease. Cell Death Dis, 2018. 9(2): p. 204.

52. Beccano-Kelly, D.A., et al., Synaptic function is modulated by LRRK2 and glutamate release is increased in cortical neurons of G2019S LRRK2 knock-in mice. Front Cell Neurosci, 2014. 8: p. 301.

440 53. Matikainen-Ankney, B.A., et al., Altered Development of Synapse Structure and Function in Striatum Caused by Parkinson's Disease-Linked LRRK2-G2019S Mutation. J Neurosci, 2016. 36(27): p. 7128-41.

54. Kupferschmidt, D.A., et al., Parallel, but Dissociable, Processing in Discrete Corticostriatal Inputs Encodes Skill Learning. Neuron, 2017. 96(2): p. 476-489 e5.

55. Shiotsuki, H., et al., A rotarod test for evaluation of motor skill learning. J Neurosci Methods, 2010. 189(2): 
56. Monville, C., E.M. Torres, and S.B. Dunnett, Comparison of incremental and accelerating protocols of the rotarod test for the assessment of motor deficits in the 6-OHDA model. J Neurosci Methods, 2006. 158(2): p. 219-23.

57. Rozas, G., et al., The overall rod performance test in the MPTP-treated-mouse model of Parkinsonism. J Neurosci Methods, 1998. 83(2): p. 165-75.

58. Beeler, J.A., et al., Dopamine-dependent motor learning: insight into levodopa's long-duration response. Ann Neurol, 2010. 67(5): p. 639-47.

59. Yin, H.H., et al., Dynamic reorganization of striatal circuits during the acquisition and consolidation of a skill. Nat Neurosci, 2009. 12(3): p. 333-41.

455 60. Costa, R.M., D. Cohen, and M.A. Nicolelis, Differential corticostriatal plasticity during fast and slow motor skill learning in mice. Curr Biol, 2004. 14(13): p. 1124-34.

61. Giesert, F., et al., The pathogenic LRRK2 R1441C mutation induces specific deficits modeling the prodromal phase of Parkinson's disease in the mouse. Neurobiol Dis, 2017. 105: p. 179-193.

62. Dzamko, N., et al., Inhibition of LRRK2 kinase activity leads to dephosphorylation of Ser(910)/Ser(935), disruption of 14-3-3 binding and altered cytoplasmic localization. Biochem J, 2010. 430(3): p. 405-13.

63. Lis, P., et al., Development of phospho-specific Rab protein antibodies to monitor in vivo activity of the LRRK2 Parkinson's disease kinase. Biochem J, 2018. 475(1): p. 1-22.

64. Brandon, E.P., et al., Defective motor behavior and neural gene expression in RIlbeta-protein kinase A mutant mice. J Neurosci, 1998. 18(10): p. 3639-49.

465 65. Kheirbek, M.A., et al., Adenylyl cyclase type 5 contributes to corticostriatal plasticity and striatumdependent learning. J Neurosci, 2009. 29(39): p. 12115-24.

66. Parisiadou, L., et al., LRRK2 regulates synaptogenesis and dopamine receptor activation through modulation of PKA activity. Nat Neurosci, 2014. 17(3): p. 367-76.

67. Piccoli, G., et al., LRRK2 controls synaptic vesicle storage and mobilization within the recycling pool. J Neurosci, 2011. 31(6): p. 2225-37.

68. Matta, S., et al., LRRK2 controls an EndoA phosphorylation cycle in synaptic endocytosis. Neuron, 2012. 75(6): p. 1008-21. 
69. Pan, P.Y., et al., Parkinson's Disease-Associated LRRK2 Hyperactive Kinase Mutant Disrupts Synaptic Vesicle Trafficking in Ventral Midbrain Neurons. J Neurosci, 2017. 37(47): p. 11366-11376.

475 70. Nguyen, M. and D. Krainc, LRRK2 phosphorylation of auxilin mediates synaptic defects in dopaminergic neurons from patients with Parkinson's disease. Proc Natl Acad Sci U S A, 2018. 115(21): p. 5576-5581.

71. Seol, W., D. Nam, and I. Son, Rab GTPases as Physiological Substrates of LRRK2 Kinase. Exp Neurobiol, 2019. 28(2): p. 134-145.

72. Kang, U.J., P. Auinger, and E.I. Parkinson Study Group, Activity enhances dopaminergic long-duration 480 response in Parkinson disease. Neurology, 2012. 78(15): p. 1146-9.

73. Zhuang, X., P. Mazzoni, and U.J. Kang, The role of neuroplasticity in dopaminergic therapy for Parkinson disease. Nat Rev Neurol, 2013. 9(5): p. 248-56.

74. Koranda, J.L., et al., Chronic Nicotine Mitigates Aberrant Inhibitory Motor Learning Induced by Motor Experience under Dopamine Deficiency. J Neurosci, 2016. 36(19): p. 5228-40.

485 75. Nandhagopal, R., et al., Progression of dopaminergic dysfunction in a LRRK2 kindred: a multitracer PET study. Neurology, 2008. 71(22): p. 1790-5.

76. Helmich, R.C., et al., Reorganization of corticostriatal circuits in healthy G2019S LRRK2 carriers. Neurology, 2015. 84(4): p. 399-406.

77. Vilas, D., et al., Clinical and imaging markers in premotor LRRK2 G2019S mutation carriers. Parkinsonism Relat Disord, 2015. 21(10): p. 1170-6.

\section{Figure Legends}

Figure 1. RC andGS LRRK2 mice have decreased nigrostriatal dopamine release.

a. A schematic diagram of the LRRK2 protein. A family of a Ras-like G-proteins with functionally distinct multi495 domains, consisting of armadillo repeats (ARM), ankyrin repeats (ANK), the leucine-rich repeats (LRR), the ROC GTPase domain where the R1441C/G/H mutations reside, and the kinase domain where the G2019S mutation resides. The WD40 domain is involved in membrane binding with ARM and ANK believed to stabilize the electrostatic surfaces of the domains. b. Brightfield photomicrograph of a parasagittal slice with a concentric 
electrical electrode placed into the dorsal striatum; a carbon fiber electrode (CFE) was inserted adjacent to the 500 stimulation site. c. Population data showing the peak evoked [DA] in WT ( $n=13$ recordings), GS ( $n=19$ recordings), and RC ( $n=19$ recordings) LRRK2 KI mice. $\mathbf{d}$. Representative time courses for [DA] with corresponding cyclic voltammograms and color maps of fast-scanning cyclic voltammetry recordings. Colormaps are normalized to the oxidation current from the WT recording. ${ }^{\star \star} p<0.01$

\section{Figure 2. iSPNs in RC LRRK2 mice have decreased excitability.}

a-b. Representative whole-cell current-clamp recordings of dSPNs and iSPNs in WT and RC LRRK2 KI mice. The three injection steps shown elicited the voltage responses to first hyperpolarizing current, the first occurrence of action potentials, and the maximum firing, respectively. The half-maximum firing values in the population data of WT mice for dSPNs ( $=475$ pA, $n=20$ recordings $)$ and iSPNs ( $=325$ pA, $n=20$ recordings $)$ were used for

510 statistical comparison with the population F-I data of RC LRRK2 KI mice for dSPNs ( $\mathrm{n}=30$ recordings) and iSPNS ( $n=23$ recordings). The dashed line represents the current where the maximal difference between the F-I functions for the iSPNs in WT and RC mice was calculated $(I=625$ pA). c-d. Representative whole-cell current-clamp recordings and population data for SPNs of WT and GS LRRK2 KI mice. The half-maximum firing values in the population data ofWT for dSPNs ( $=375 \mathrm{pA}, \mathrm{n}=13$ recordings $)$ and iSPNs $(\mathrm{I}=325 \mathrm{pA}, \mathrm{n}=20$ recordings $)$ were 515 used for statistical comparison with the population F-I data of GS LRRK2 KI mice for dSPNs ( $\mathrm{n}=29$ recordings) and iSPNs ( $n=28$ recordings). F-I data are shown as mean \pm standard error of the mean. $* \star \star ~ p<0.001$

\section{Figure 2-figure supplement 1. iSPNs have increased membrane capacitance in RC LRRK2 mice.}

a. Scatter plots of membrane capacitance with medians (center line) and interquartile ranges (whiskers) for WT 520 dSPNS ( $n=25$ recordings) and iSPNS ( $n=27$ recordings) and RC LRRK2 KI dSPNs ( $n=38$ recordings) and iSPNs ( $n=29$ recordings). b. Scatter plots of membrane capacitance for SPNs in both WT and GS LRRK2 KI mice. 


\section{Figure 2-figure supplement 2. iSPNs have an increased EPSC decay time in RC LRRK2 mice.}

a. Representative brightfield photomicrograph of a WT dSPN in a parasagittal slice voltage-clamped at $-80 \mathrm{mV}$. $\mathbf{b}$.

525 Spinning-disk confocal photomicrograph of the same dSPN in the panel to the left. Alexa Fluor 647 was loaded into the cell (via the patch pipette) for visualization. SPNs were identified by morphology and a large number of dendritic spines. Inset: magnified view of the boxed region showing a dendritic segment that is heavily decorated with spines. c. Top left: Representative voltage-clamp recording of the dSPN shown in b above. Paired-pulse stimulation of the cortex at $20 \mathrm{~Hz}$ elicited a pair of excitatory postsynaptic currents (EPSC). Bottom left: Magnified 530 view of the decay of the second EPSC shown in the boxed region above. The red portion depicts the $95 \%-5 \%$ decay time. $\mathbf{d}$. Population data of paired-pulse ratios of WT dSPNs ( $n=14$ recordings) and iSPNs $(n=13$ recordings) and $\mathrm{RC}$ dSPNs ( $\mathrm{n}=17$ recordings) and iSPNs ( $\mathrm{n}=18$ recordings). e. Population data of the $95 \%-5 \%$ decay times of SPNs from age-matched WT dSPNs ( $n=14$ recordings) iSPNs ( $n=13$ recordings) and RC dSPNs ( $n=13$ recordings) iSPNs ( $n=18$ recordings).

535

\section{Figure 3. RC KI mice show dopamine-dependent motor learning impairments.}

a. Schematic of the rotarod training paradigm. Mice were assessed over a total of 18 daily sessions where every daily session consisted of five trials. WT, GS, and RC mice were administered either saline or a cocktail of D1 receptor (SCH23390) and D2 receptor (eticlopride), $1 \mathrm{mg} / \mathrm{kg}$ of each antagonist $30 \mathrm{~min}$ prior to daily sessions, and 540 trained for five successive days in an accelerated rotarod. After a $72 \mathrm{~h}$ break, the mice were returned to the rotarod for an additional 13 days of a drug-free recovery phase. b. Genotype-dependent effect of blocking both D1 and D2 receptors, during the first five sessions on rotarod performance of WT, RC, and GS mice. The average latency in the early (session 6-8) and late (session 16-18) stage in the drug-free recovery phase in b is summarized in c. Open and filled bars represent the average performance of saline control and drug-treated mice, respectively 545 (saline treated groups: $n_{W T}=10$ mice, $n_{R C}=10$ mice, and $n_{G S}=9$ mice; $D 1+D 2$ receptor antagonist treated groups: $\mathrm{n}_{W T}=13$ mice, $\mathrm{n}_{\mathrm{RC}}=11$ mice, and $\mathrm{n}_{\mathrm{GS}}=11$ mice; ${ }^{\star \star \star} p<0.001$ vs genotype-matched saline control). $\mathbf{d}$. The motor learning deficit induced by dopamine antagonism was not observed in RC mice pre-treated with the LRRK2 inhibitor MLi-2, which was administered daily, 60 min prior to training. group of RC mice that were then treated 
with either saline (open circles) or antagonist cocktail (closed circles) for the first 5 sessions. Dash and solid lines

550 respectively represent the saline control or dopamine receptor antagonist treated groups. Inset shows a decrease in S935 LRRK2 phosphorylation, which reflects LRRK2 kinase activity in WT mice after 60 min Mli-2 administration. e. Average latency in blocks of 3 sessions during the drug free phase from $\mathbf{d}$. MLi-2 with saline: $n=12$ mice; MLi2 with D1+D2 antagonists: $\mathrm{n}=11$ mice; ${ }^{\star \star} p<0.01$ ).

\section{Figure 3-figure supplement 1. Effect of D1 or D2 antagonism on dopamine-dependent motor learning.}

Rotarod performance of WT, RC, and GS mice that were administered with an antagonist for either D1 (SCH23390) (a-b) or D2 receptors (eticlopride) (c-d), 30 min prior to training for the first five days. Solid traces are saline control replotted from Figure 3b for reference. $\mathbf{b}$ and $\mathbf{d}$. Averaged latency of saline control (open bars) and drugtreated mice (filled bars). Early and late refer to session 6-8 and 16-18, respectively, of the no drug recovery phase 560 (D1R antagonist treated groups: $n_{W T}=11, n_{R C}=11$, and $n_{G S}=9$ mice; D2R antagonist treated groups: $n_{W T}=9, n_{R C}=$ 14 , and $\mathrm{n}_{\mathrm{GS}}=10$ mice; ${ }^{\star \star \star} p<0.001,{ }^{\star \star} p<0.01$ vs genotype-matched saline control). e. Improved performance of $\mathrm{RC}$ mice received antagonist cocktail but no training during the first five sessions. The $\mathrm{RC}_{\mathrm{D} 1+\mathrm{D} 2}$ group data are from Figure $\mathbf{3 b}$ for reference (stats). f. Distance travelled in the open field after five days of rotarod training with either vehicle or dopamine receptor antagonist cocktail treatment. Another group of mice received dopamine antagonist

565 treatment and rotarod training for five days under the same schedule described in $\mathbf{a}-\mathbf{d}$ and Figure $\mathbf{3 b} \mathbf{b}$, and were tested in the open field immediately after the 72-hr break instead of the drug-free phase (saline treated groups: nwT $=11$ mice, $n_{R C}=8$, and $n_{G S}=7$ mice; $D 1 R+D 2 R$ antagonist treated groups: $n_{W T}=7, n_{R C}=6$, and $n_{G S}=6$ mice; D1R antagonist treated groups: $\mathrm{n}_{W T}=7, \mathrm{n}_{\mathrm{RC}}=9$, and $\mathrm{n}_{\mathrm{GS}}=9$ mice; $\mathrm{D} 2 \mathrm{R}$ antagonist treated groups: $\mathrm{n}_{W T}=8, \mathrm{n}_{\mathrm{RC}}=11$, and $\mathrm{n}_{\mathrm{GS}}=7$ mice)

Figure 3-table supplement 1. Summary of differences between mutants and pharmacological conditions.

Summary table of $p$-values for within-treatment and within-genotype post hoc comparisons for both the early and late sessions during the drug-free recovery phase. 
a

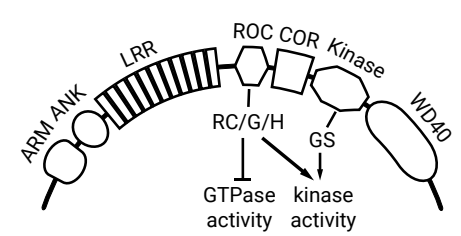

d
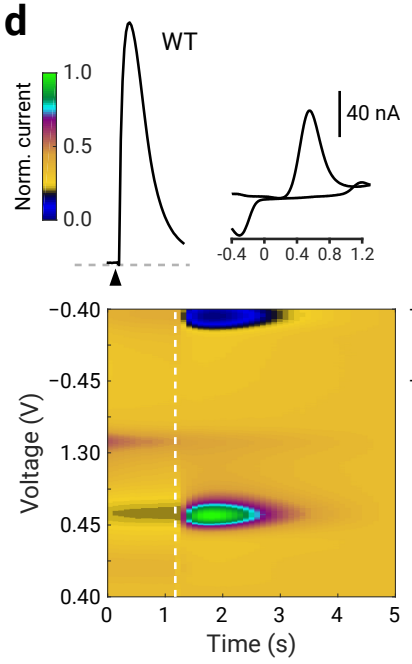

b

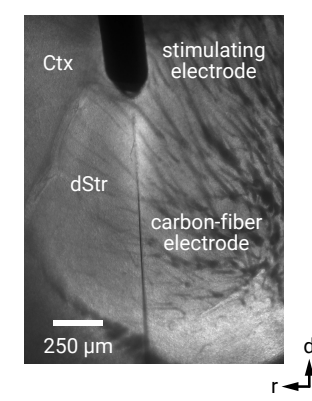

$\mathrm{RC}$

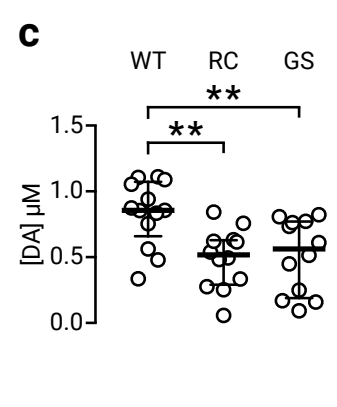

GS
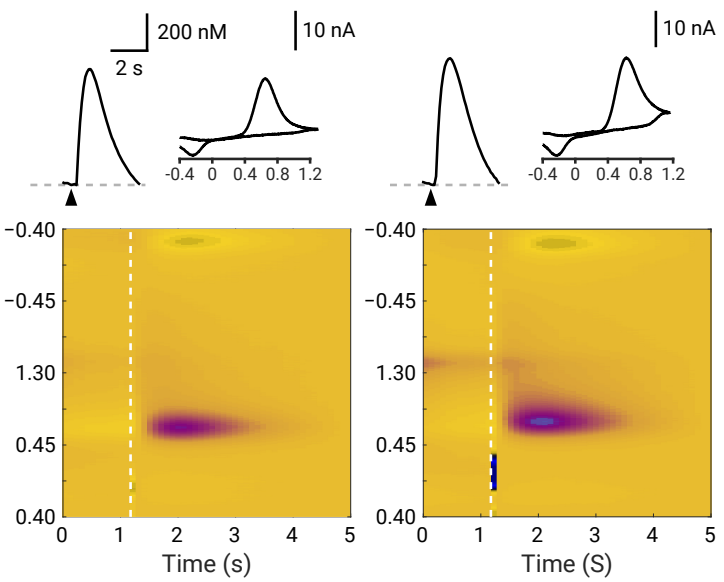

Figure 1 
bioRxiv preprint doi: https://doi.org/10.1101/2020.03.11.986455; this version posted July 29, 2020. The copyright holder for this preprint (which was not certified by peer review) is the author/funder. All rights reserved. No reuse allowed without permission.
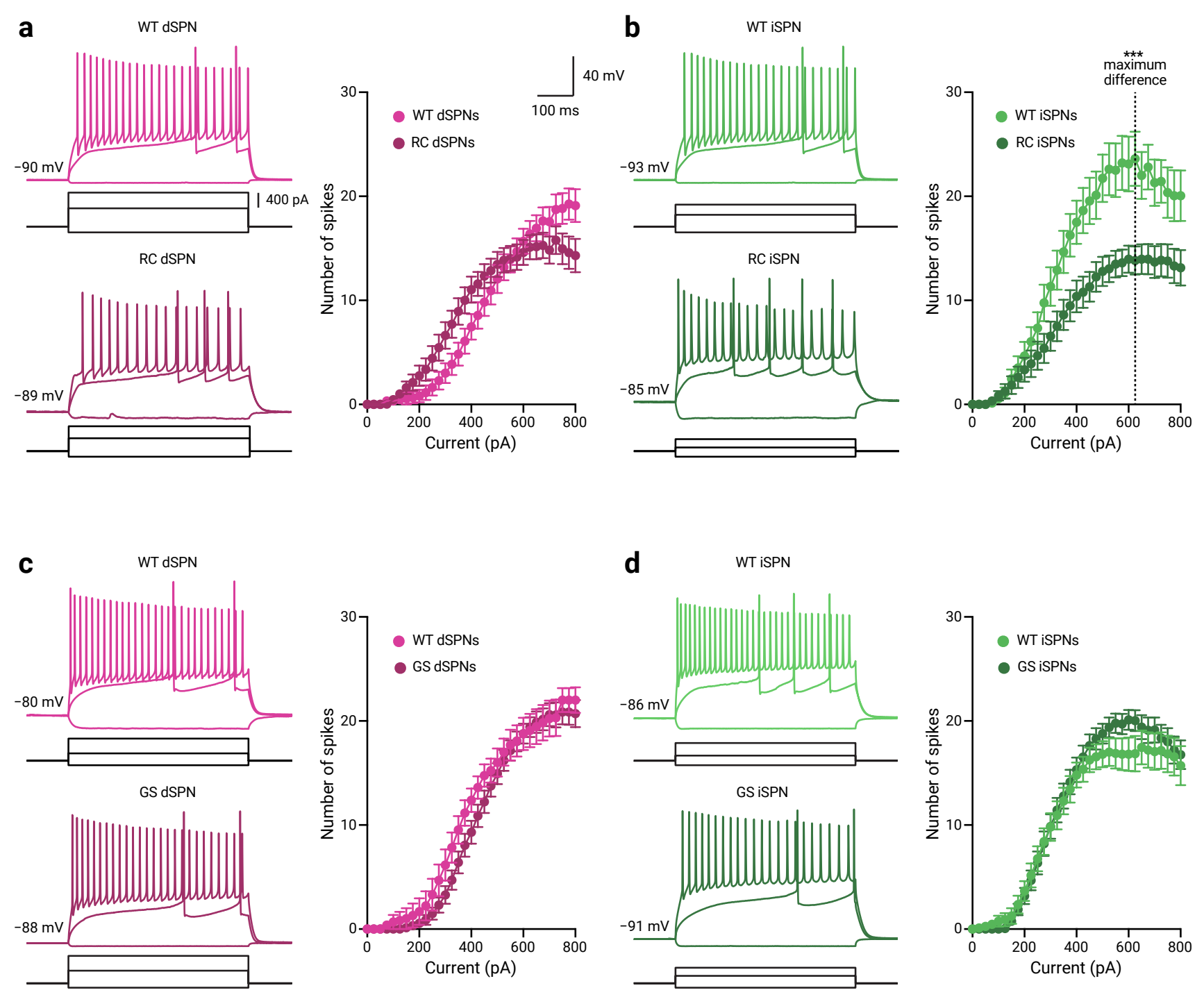

Figure 2 


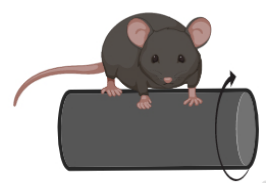

\begin{tabular}{|c|c|c|}
\hline $\begin{array}{c}\mathrm{D} 1+\mathrm{D} 2 \\
\text { antagonism }\end{array}$ & $\begin{array}{l}72 \mathrm{hr} \\
\text { break }\end{array}$ & $\begin{array}{l}\text { Drug-free } \\
\text { recovery }\end{array}$ \\
\hline Session 1-5 & & Session 6-18 \\
\hline
\end{tabular}

b

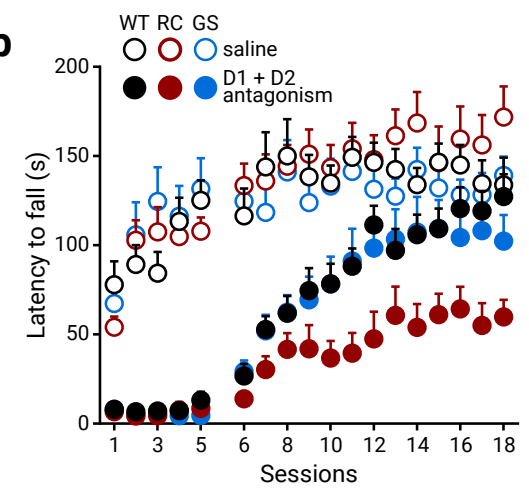

veh MLi-2

d

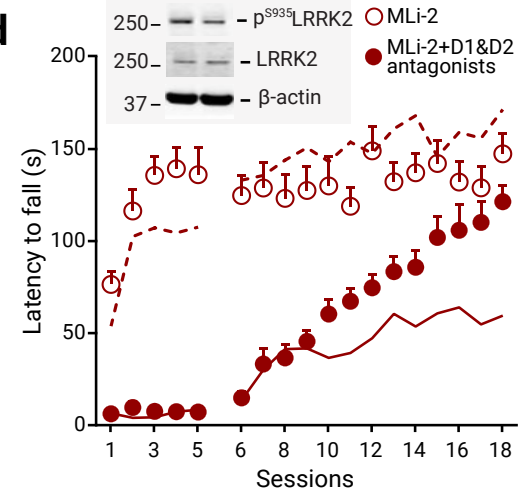

C

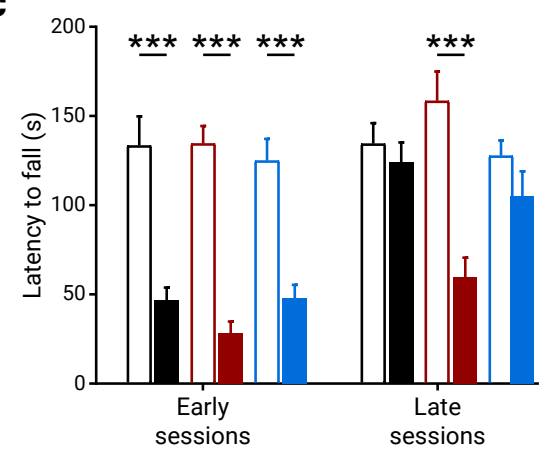

e

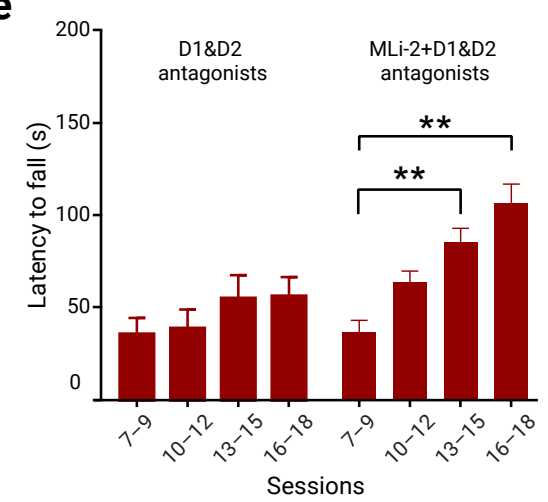

Figure 3 
a

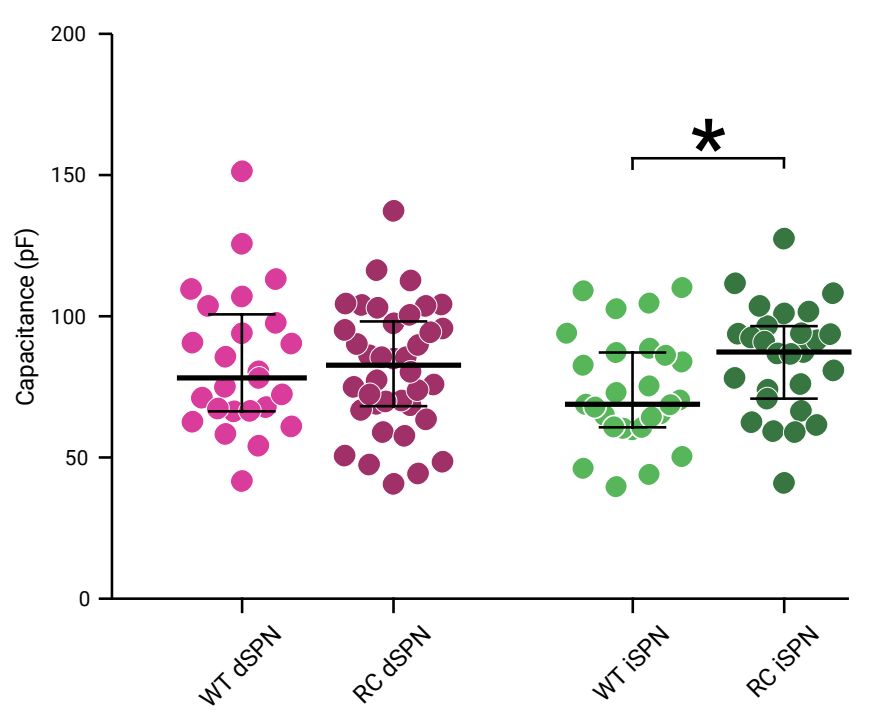

b

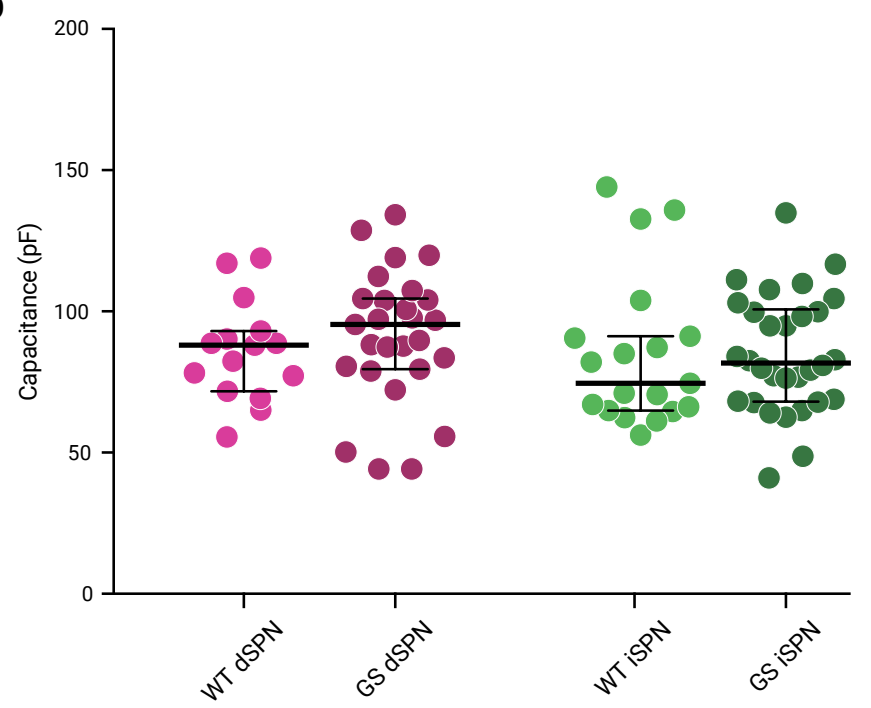

Figure 2-figure supplement 1 

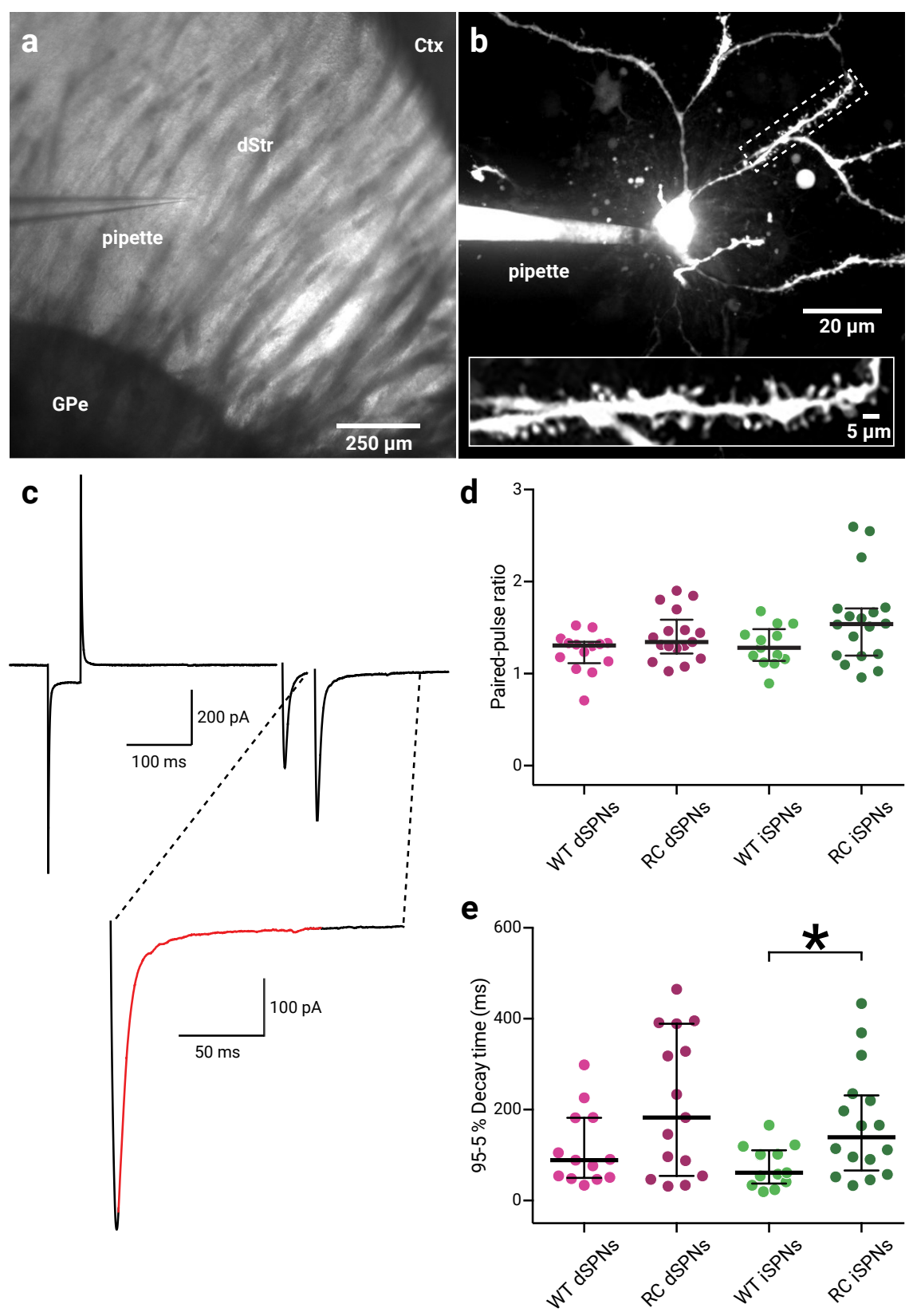

Figure 2-figure supplement 2 

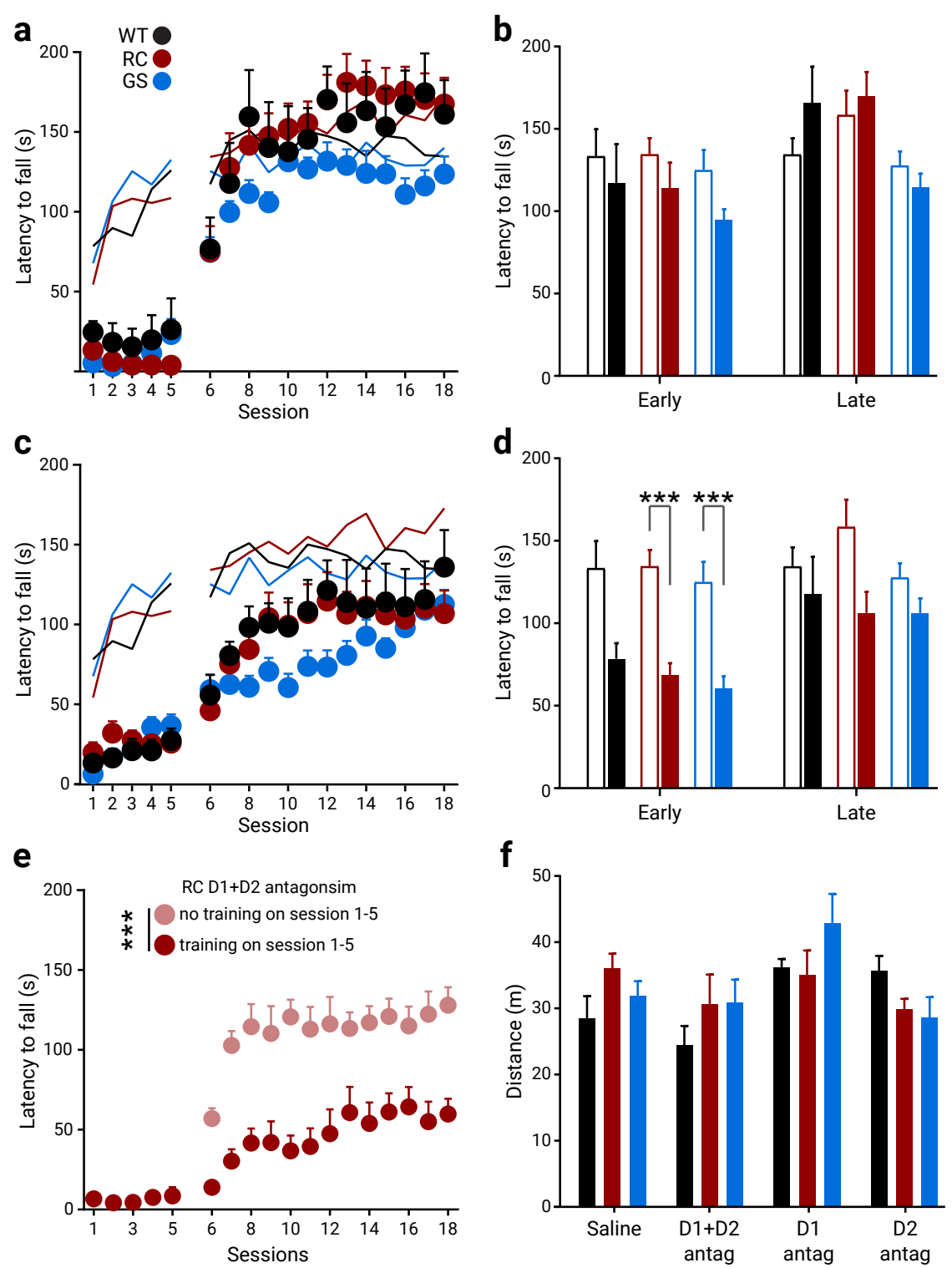

Figure 3-figure supplement 1 


\section{Early sessions 6-9}

Saline vs. D1+D2 antagonism Saline vs. D1 antagonism Saline vs. D2 antagonism

\begin{tabular}{c} 
WT \\
\hline$<0.0001$ \\
0.9913 \\
0.0549
\end{tabular}

D1 + D2 antagonism

WT vs. RC WT vs. GS

GS vs. RC

\begin{tabular}{c} 
RC \\
\hline$<0.0001$ \\
0.9593 \\
0.0190
\end{tabular}

D1 antagonism 1.0000 0.9781 0.9936
D1

$\begin{array}{ccc}\text { antagonism } & & \text { Saline } \\ 1.0000 & & 1.0000 \\ 0.9981 & & 1.0000 \\ 1.0000 & & 1.0000\end{array}$

Late sessions $16-18$

Saline vs. D1+D2 antagonism

Saline vs. D1 antagonism Saline vs. D2 antagonism

\section{WT}

0.9999

0.5480

1.0000

D1 + D2 antagonism

WT vs. RC

WT vs. GS

GS vs. RC
0.0429

0.0952

0.4980
D1 antagonism

1.0000

0.3641

0.2661

\section{RC} $<0.0001$

1.0000

0.1492

D1

\begin{tabular}{c} 
antagonism \\
\hline 1.0000 \\
1.0000 \\
1.0000
\end{tabular}

GS

0.0007

0.7990

0.0128

90
28

\section{S}

